\title{
A Cross-Sectional Study Evaluating Potential Differences in the Need for Cancer Survivorship Care in Relation to Patients' Socioeconomic Status
}

\author{
Charlotte Handberg, b, c, d, Marie Louise Svendsen ${ }^{b}$, Thomas Maribo ${ }^{a}$, b
}

\begin{abstract}
Background: There seems to be inequity within cancer survivorship care in primary care settings related to gender, shorter education, and early poor health, but there is uncertainty regarding the character of the needs in hospital and in primary care settings and whether there is inequity regarding meeting these needs. This study aims to describe potential differences in needs among patients in hospital and in primary care settings, and to assess the need for survivorship care and rehabilitation in patients with cancer in relation to socioeconomic status.
\end{abstract}

Methods: In a cross-sectional study including patients in hospital (n $=89)$ and primary care settings $(\mathrm{n}=99)$, information from needs assessments was linked with population-based data on socioeconomic status via unique personal identification numbers. The association between socioeconomic status and stated needs was analyzed separately for patients in hospital and primary care settings, with adjustment for age and gender.

Results: A total of $90 \%$ patients expressed one or more needs in the physical area, $51 \%$ in the emotional area, and $40 \%$ in the practical area. Patients in primary care expressed more needs than patients in hospital. Men expressed more needs than women in primary care (adjusted odds ratio (OR) (95\% confidence interval (CI)): 2.66 (1.04 - 6.79)). The results indicate that higher socioeconomic status is associated with fewer stated needs.

Conclusions: This study suggests that the association between gender and stated needs may depend on healthcare setting and confirms that higher socioeconomic status in relation to civil status, educational level, income, and labor market status is associated with fewer stated needs.

Manuscript submitted May 7, 2019, accepted May 29, 2019

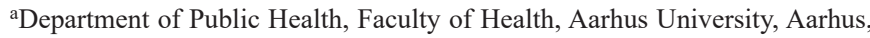
Denmark

bDEFACTUM, Central Denmark Region, Aarhus, Denmark

'The Danish National Rehabilitation Center for Neuromuscular Diseases, Aarhus, Denmark

${ }^{\mathrm{d} C o r r e s p o n d i n g ~ A u t h o r: ~ C h a r l o t t e ~ H a n d b e r g, ~ D e p a r t m e n t ~ o f ~ P u b l i c ~ H e a l t h, ~}$ Faculty of Health, Aarhus University, Kongsvang Alle 23, Aarhus 8000, Denmark.Email: chha@rcfm.dk

doi: https://doi.org/10.14740/jocmr3871
Keywords: Cancer; Survivorship care; Survivorship; Needs; Socioeconomic status; Cross-sectional

\section{Introduction}

The number of cancer survivors in Europe is estimated to be over 18 million in 2017, which calls for public health policies defining and assigning services for the survivors [1]. It is roughly estimated that around $70 \%$ of patients with cancer have potential needs for survivorship care after treatment [2, 3]. However, the number of patients participating in cancer survivorship care (within which we include rehabilitation) in the primary care services in Denmark is much lower than the number of patients who actually have need for survivorship care $[4,5]$. A large group of patients thereby miss the opportunity to optimize physical, psychological, vocational, and social functioning through survivorship care, which would help to counteract the limitations imposed by the side effects of cancer treatments and/or comorbid conditions [5-8].

For the most part, public health systems are based on the citizens' self-management, like being actively engaged in own treatment and survivorship care and contacting the health system when in need [9]. This organization, however, calls for citizens with surplus energy, and could lead to groups of patients not seeking information or being informed of the possibility for and effect of cancer survivorship care, with the result that they miss out on survivorship care [5, 10-12]. This kind of inequity is argued to be present within cancer survivorship care, since the most exposed patients apparently do not participate in survivorship care in primary care services to the same extent as other patients $[4,11,12]$.

Assessing patients' needs for survivorship care is an initial and essential step in the path to secure survivorship care for the patients in need $[4,5]$, thereby reducing inequity in cancer care by also ensuring exposed groups of patients to receive the benefits of cancer survivorship care [13-15]. In Denmark, health agreements have been made which ensure that all patients with cancer receive a needs assessment $[16,17]$. These agreements are meant to ensure that patients assessed to be in need at hospital are referred to the primary care survivorship care programme. Despite a well-described and defining public health policy among healthcare sectors, not all patients receive 
a needs assessment, and the procedures around the needs assessment are apparently inconsistent and not formalized [5, 18]. There seems to be inequity within cancer survivorship care in primary care settings related to gender, shorter education, and early poor health [12], but there is uncertainty regarding the character of the needs in hospital and in primary care settings and whether there is inequity with regard to meeting these needs.

In this study, we aim to describe potential differences in needs among patients in hospital and in primary care settings, and to assess the need for survivorship care and rehabilitation in patients with cancer in relation to socioeconomic status.

\section{Materials and Methods}

\section{Design}

This cross-sectional study included patients with a cancer diagnosis, aged 18 years or older, who had a needs assessment conducted between April 2015 and December 2015 in two specific hospital departments or in two cancer survivorship care settings in primary care. Data from the needs assessments were linked with patient-level clinical and socioeconomic information from nationwide population-based medical and social registries through a unique personal identification number. Since 1968, all Danish residents have been assigned a unique 10-digit personal identification number, which is used in all databases and allows for explicit person-level linkage between databases [19].

\section{Setting}

In Denmark, the healthcare system is divided into two sectors with five regions and 98 municipalities. The regions are responsible for all hospital service plus a needs assessment after a hospital stay and referral to survivorship care. The primary care settings are responsible for conducting survivorship care. The two included hospitals have a background population of 1.3 million people, whereas the two primary care settings represent a shared background population of 390,000 individuals.

\section{Participants}

The study included a convenience sample of hematological cancer patients from two hospital departments of hematology $(n=89)$ and patients with all cancer types from two primary care settings offering survivorship care $(n=99)$. Patients were eligible to participate if they had a cancer diagnosis, were aged 18 or older, and had a needs assessment conducted between April 2015 and December 2015.

\section{Needs assessment}

The needs assessment form assigned by the Central Denmark
Region consists of six domains and 58 fixed areas to identify and state possible needs (Supplementary Figure 1) [5]. A tick in either "area" or "need for support" (or both) was registered as one stated need, meaning each patient could potentially state 0 to 58 needs [5]. All patients cooperated in the assessment of their survivorship care needs with a healthcare professional (a nurse, a physiotherapist, or a dietician) [5].

\section{Clinical and socioeconomic characteristics}

Information on gender, age, cohabitation status, highest attained education, income, and labor market status was obtained from nationwide population-based registers on education, income, and labor market affiliation [20-22]. Data were obtained for the most recent date of registration prior to the date of the needs assessment. These registers are continually updated at least once a year, and the validity and coverage of the registers are high [20-22]. Information on cancer diagnoses was obtained from the Danish Cancer Register, which contains validated and complete diagnoses of all incident cancers in Denmark since 1943 [23]. The diagnoses are coded according to the 10th revision of the International Classification of Disease (ICD-10) since 1978 (according to the ICD-7 from 1943-1977).

\section{Statistical analysis}

A total needs score was calculated by summing each patient's needs across the six domains of stated needs (range 0 - 58) (Supplementary Figure 1). The mean number of stated needs per patient was eight, and the total needs score was categorized accordingly (i.e. 0 - 7 needs vs. $8+$ needs). When analyzing each domain of stated need, the domain-specific needs scores were categorized as 0 stated needs versus one or more stated needs (Supplementary Figure 1) [5]. The clinical and socioeconomic data were classified as shown in Table 1 except that age was included as a discrete co-variable in the adjusted analyses [11].

Associations between socioeconomic status and stated needs were analyzed by unadjusted and adjusted logistic regression analyses and expressed as odds ratios (ORs) and 95\% confidence intervals (CIs). All analyses were performed separately for patients in hospital and in primary care settings to evaluate potential differences between the two care settings, and adjustment was made for age and gender [12]. A sensitivity analysis was performed that excluded patients with breast cancer, as patients with breast cancer represent a large proportion in primary care and because women with higher socioeconomic status have higher risk of breast cancer [24]. All analyses were performed in Stata 15 , with a $\mathrm{P}$ value $<0.05$ being considered statistically significant.

\section{Ethical considerations}

This study was conducted in compliance with the ethical stand- 
Table 1. Sociodemografic Characteristics Among Patients With Cancer in Hospital and Primary Care

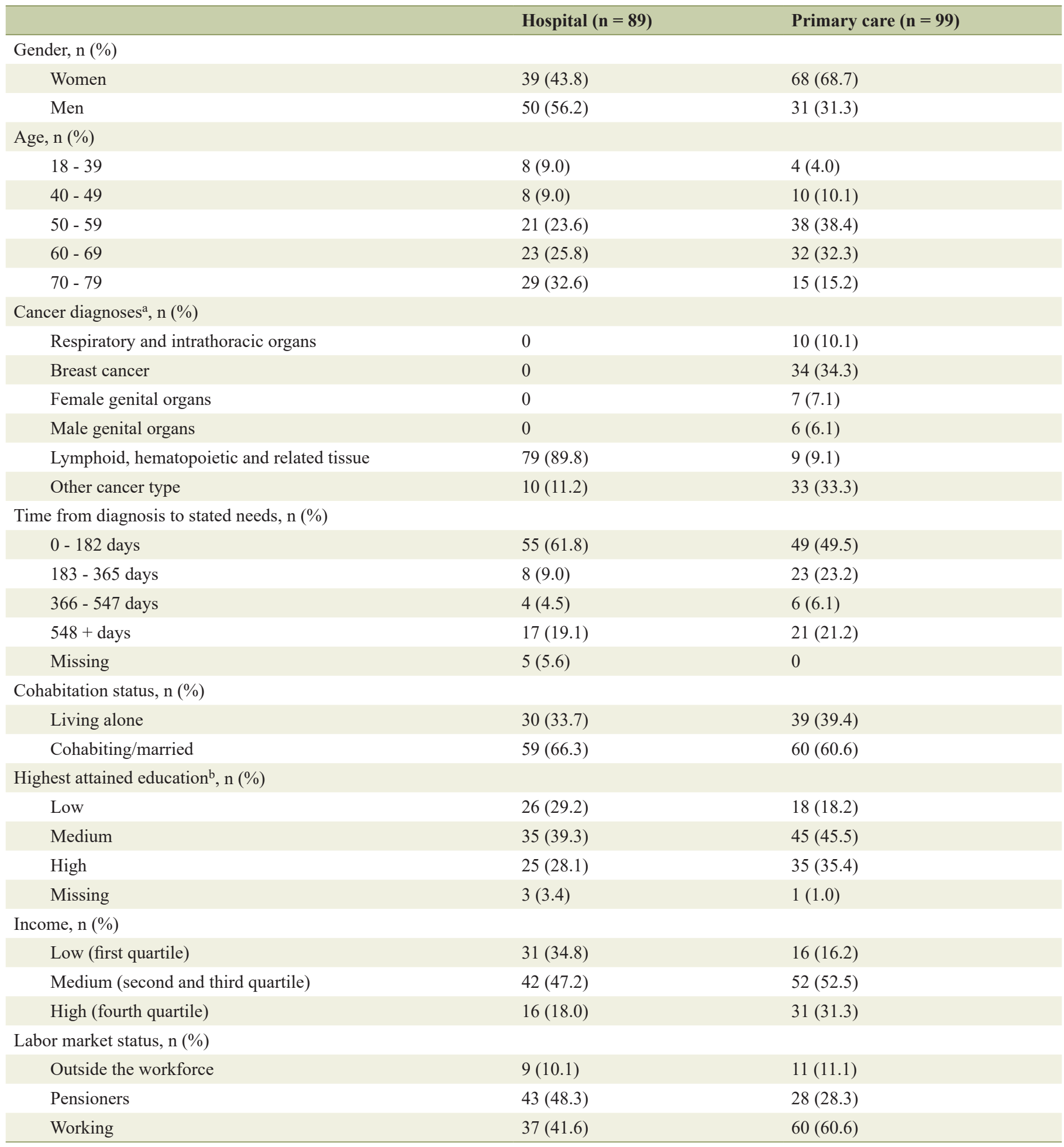

aOther cancer types include cancer of lip, oral cavity and pharynx, digestive organs, melanoma and other malignant neoplasms of the skin, mesothelial and soft tissue, urinary tract, thyroid and endocrine glands, as well as cancer of ill-defined, secondary and unspecified sites; bLow (primary school), medium (upper secondary school, vocational education), and high (short-, medium- and high-term higher education and PHD).

ards of the responsible institution on human subjects as well as with the Helsinki Declaration. Ethical approval was obtained from the Danish Data Protection Agency approval (number 2007-58-0010). Eligible patients were invited to participate in 
the project, and both oral and written consents were given.

\section{Results}

A total of 188 patients with cancer, 89 at two hospitals, and 99 in two primary care settings, completed a needs assessment form. Baseline characteristics are displayed in Table 1. Compared with patients in hospital, patients in primary care were more often women ( $69 \%$ vs. $44 \%)$, middle-aged $(71 \%$ vs. $49 \%$ ), had a medium to high level of education ( $81 \%$ vs. $67 \%$ ), a medium to high income ( $84 \%$ vs. $65 \%$ ), and were in the workforce $(61 \%$ vs. $42 \%)$. Patients in primary care had diverse cancer diagnoses, with breast cancer constituting the largest part (34\%), whereas the majority of patients in hospital suffered from hematological cancer $(90 \%)$. A total of $50 \%$ and $62 \%$ of the patients in primary care and hospital, respectively, completed the needs assessment within 6 months after diagnosis.

Table 2 shows that men express more stated needs compared with women in primary care (adjusted OR $(95 \% \mathrm{CI})$ : $2.66(1.04-6.79))$, but this association between gender and needs is not found when the analysis was restricted to patients in hospital (adjusted OR (95\% CI): $0.89(0.37-2.15)$ ) or when the data were pooled across primary care and hospital (adjusted OR $(95 \%$ CI): $1.30(0.71-2.39))$. Furthermore, married and cohabiting patients tended to state fewer needs than patients living alone, but the results did not reach statistical significance (adjusted OR ( $95 \%$ CI): $0.55(0.30-1.02))$. We were unable to show any associations between educational level, income, and labor market status in relation to stated needs, but all point estimates suggested that higher socioeconomic status is associated with fewer stated needs (Table 2).

Nearly all patients in hospital and in primary care stated one or more needs (Table 3). Pooling the data across primary care and hospital, the majority of the patients expressed one or more needs in the physical area $(90 \%)$ and the emotional area $(51 \%)$, and fewer patients expressed one or more needs in the practical area $(40 \%)$, the work/school area $(17 \%)$, the family area $(13 \%)$, and the spiritual/religious area $(2 \%)$. In general, patients in primary care expressed more needs than patients in hospital except for the practical areas, where patients in hospital expressed more needs than patients in primary care (Table 3 ).

The results of the sensitivity analysis that excluded patients with breast cancer $(n=34)$ support the finding that the association between gender and stated needs depends on the healthcare setting (primary care, men vs. women - adjusted OR (95\% CI): $1.32(0.44-3.98))$, that married and cohabiting patients state fewer needs than patients living alone (adjusted OR (95\% CI): $0.47(0.24-0.94)$ ), and that the remaining results point towards higher socioeconomic status in relation to educational level, income, and labor market status being associated with less stated needs.

\section{Discussion}

The fact that more women completed a needs assessment form in the primary care setting was not surprising (Table 1). Research has shown that men usually are more reluctant to express needs, feelings, or ask for support [25]. It is also known that men are less likely than women to take part in survivorship care, reasons being that they have a tendency to be less interested in initiatives related to health-like survivorship care initiates, and hence are more reluctant to seek professional help and support [26-28]. Men in general tend to live a chancier lifestyle in relation to health and cancer $[29,30]$, but research on men and health promotion has shown the importance of choosing new approaches for survivorship care [31]. New approaches outside the normal survivorship care setting or training facility can promote a situation in which men feel less threatened by the thought of talking about their cancer disease and everything that it entails [25]. Taking a systematic approach to the needs assessment and indicating that it is part of the mandatory approach to survivorship care could indicate the normality of assessing needs for survivorship care in general regardless of the gender of the patient.

Our results show that men express more needs than women in primary care (Table 2), and this tendency is confirmed when the relatively large part of women with breast cancer in primary care are excluded. This result does not relate well with the theory that men are more reluctant to express needs [26-28]. Research suggests that men with cancer may state fewer needs for further information about psychological support although they feel less informed about the topic compared with women [32]. In previous research, it has been emphasized that women are generally more at ease with expressing concerns, feelings, and needs openly, which results in health professionals often assuming that female patients have more needs than men [33, 34]. Our finding that men in primary care express more needs than women underlines the need for using a needs assessment form systematically in all patients. This might be a way to get around a possible gender inequity in relation to cancer survivorship care.

The results also indicate that cohabiting patients state fewer needs, which is consistent with the literature showing that women living alone tend to more often state needs for survivorship care and have more unmet needs [11]. Further, it is known that vulnerable women have delays in care [35], and men living alone and men outside the workforce have increased odds of having unmet needs in the physical area [11]. Our study also indicated that those patients with low socioeconomic status were also the ones who stated the most needs (Table 2). Research has shown that those with the highest attained education have a better chance of being referred to rehabilitation services [12], and our results emphasize the importance of referring patients with low socioeconomic status to cancer rehabilitation. Women, but also men with low education and low income, participate less often in services such as survivorship care in spite of more stated unmet needs [11]. This is consistent with the fact that cancer patients who belong to higher socioeconomic groups of society are generally more motivated to seek help and support, for instance, through internet support groups or survivorship care services [36, 37]. In our study, the association between higher socioeconomic status and fewer stated needs was seen both in hospital and in the primary care setting. Further, the literature has shown a clear social gradient in cancer survivors' health behaviors, which emphasizes the need for much more focus on socially differentiated initiatives 


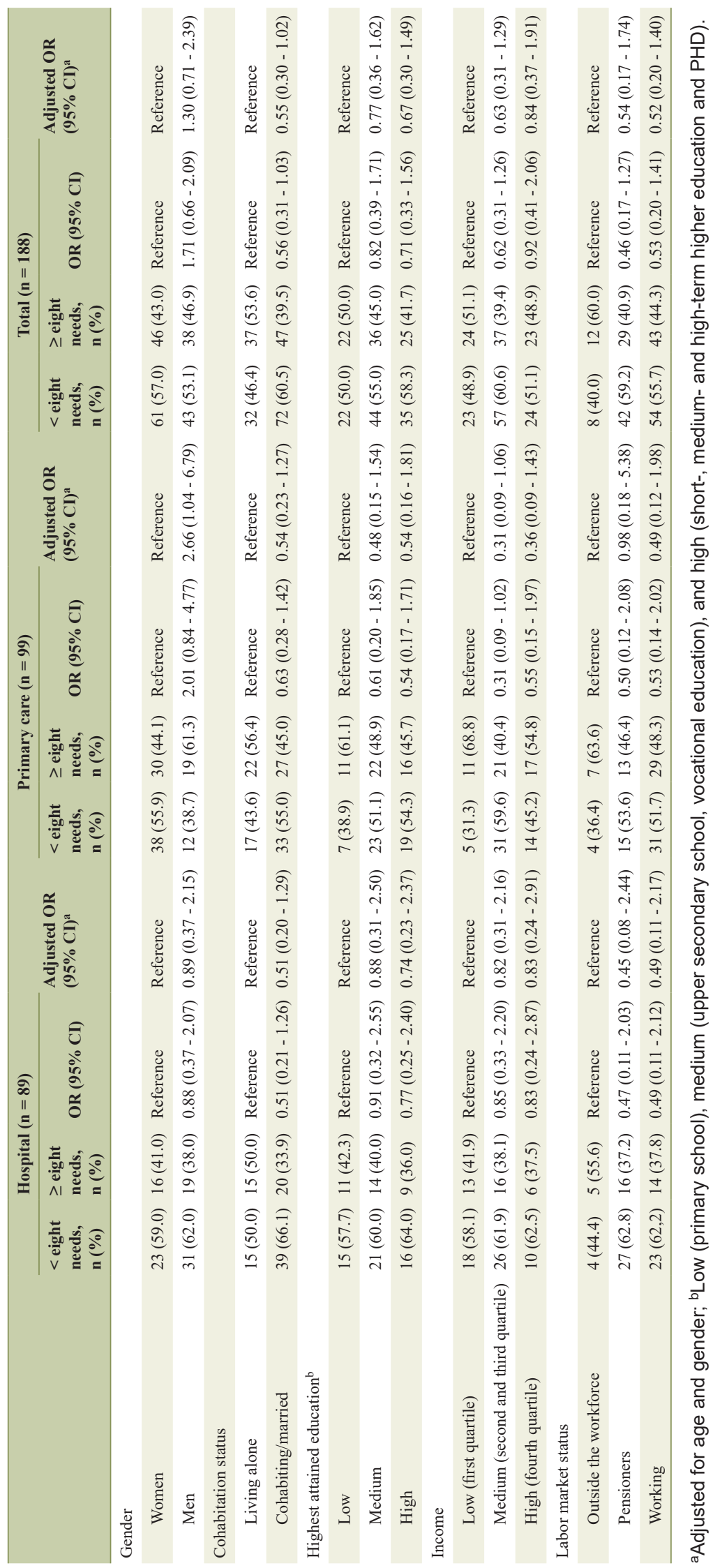


Table 3. Stated Needs Among Patients With Cancer at Hospital and in Primary Care

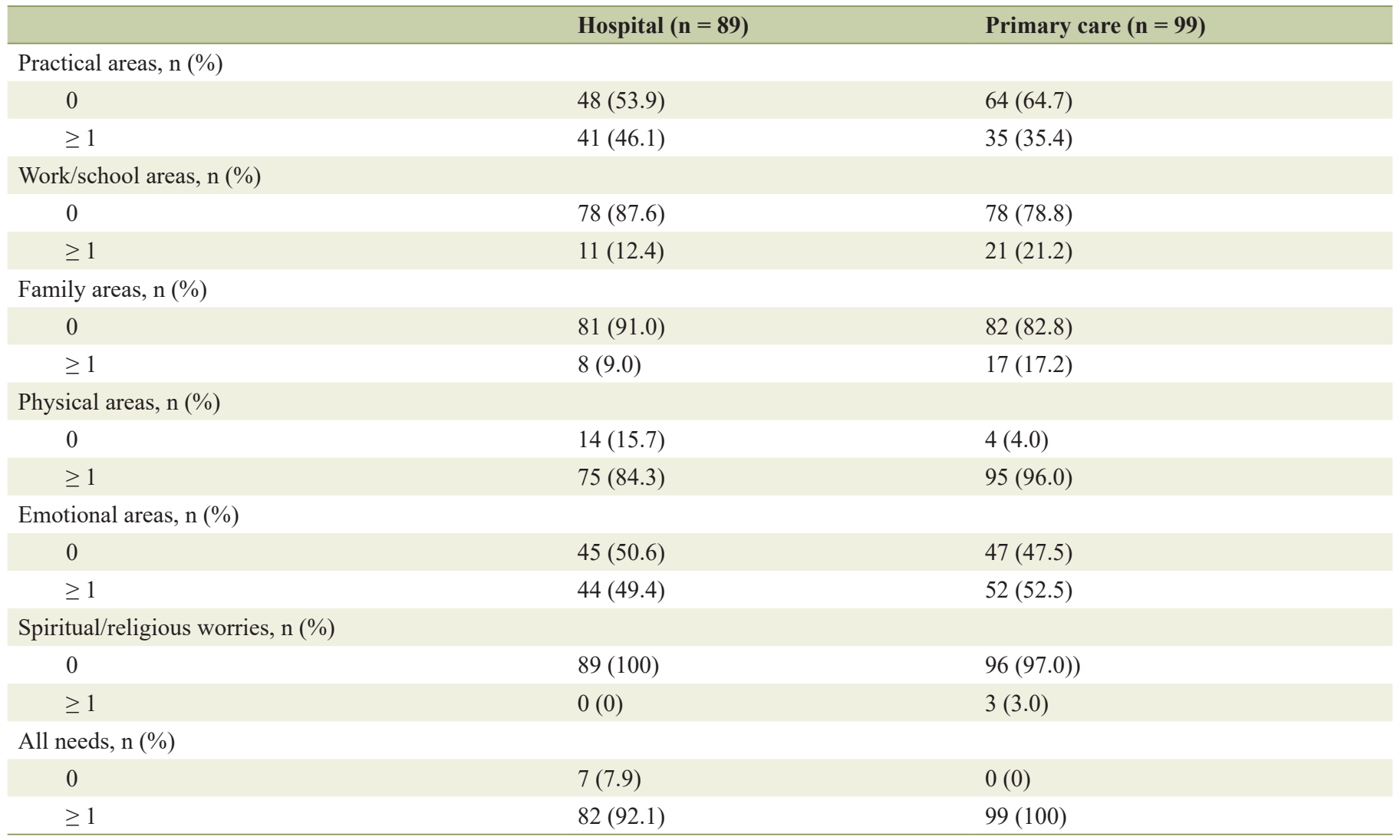

of cancer survivors [10]. In addition, we recommend special focus on socioeconomically disadvantaged groups when developing initiatives in the field of survivorship care [11]. The results emphasize the need for a systematic needs assessment in order to ensure that everyone is given the possibility for cancer survivorship care and that referral is undertaken if needed. Lastly, we found that needs related to the physical and emotional areas at hospitals and in primary care settings were more often stated than other needs (Table 3). This is consistent with findings from a prior study in which we also found that being worried was stated as the third largest need, with almost the same proportion among hospital and primary care patients [5].

We argue that these needs should be integrated into cancer survivorship services [38]. Unfortunately, there is a tendency for the services to pay more attention to the physical elements of survivorship care and forget about psychosocial needs [39]. This is unfortunate since we know survivorship care to be effective and beneficial with regard to many parameters [13, 40-42], so if we know that many patients also state emotional needs, there seems to be an important need to direct survivorship care interventions towards this need for psychological support as well as for support in the physical area [5].

\section{Strengths and limitations}

A main strength of our study is that information on socioeco- nomic status was collected via administrative records prior to the study and was independent of the needs assessment and healthcare setting. Thereby, the risk of information bias regarding socioeconomic data is reduced, and data across primary care and hospitals is made comparable. However, several different healthcare professionals collected data about patients' needs, in collaboration with the patients, which may incur inter-observer variation in data about stated needs. However, this potential inter-observer variation will most likely incur non-differential misclassification and direct the results towards a null association between socioeconomic status and stated needs.

Due to the observational nature of the study, the results may be influenced by confounding. However, we did adjust the results for age and gender, which are potentially important confounders of the association between socioeconomic status and stated needs [12]. Older age and female gender also correlate with multimorbidity [43], and therefore adjustment for age and gender may indirectly and partially account for comorbid disease as a confounder. Still, the results may be influenced by confounding factors, including disease severity, comorbid disease, and treatment modalities, which most likely would direct the results in the direction of a stronger association between lower socioeconomic status and more stated needs [44]. The bearing of these factors on the association between socioeconomic status and stated needs remains to be clarified in future research. 
It could be expected that time since diagnosis would influence the number of stated needs, which would be higher near the time the diagnosis was made, but our findings show that the number increases in the primary care setting. The finding that more needs were stated after the patients had been discharged from hospital could indicate that the patients do not realize all their survivorship care needs until they are home again in their own surroundings and in a more stable phase.

The results do not address how many patients (women and men) out of the population of eligible patients receive a needs assessment, and the study population may not be representative of all cancer patients. In particular, the patients' sociodemographic data (Table 1) and stated needs (Table 3) may differ from the total population of cancer patients, but we do believe that the associations in Table 2 are inherent and unaffected by the exact composition of patients, and that they are therefore generalizable to the population of cancer patients. This is supported by previous research $[11,45]$, showing concurrent associations between higher socioeconomic status and fewer stated needs. Still, the observational and cross-sectional design of our study implies that no firm conclusion about causal relationships can be drawn. Our study does, however, emphasizes that healthcare professionals should pay special attention to assessing needs in patients who belong to the lower socioeconomic groups of society, and to male cancer patients in primary care $[11,45]$, which was substantiated by prior research.

\section{Conclusions}

This is the first study to assess needs for survivorship care in patients with cancer in relation to socioeconomic status at hospital and in primary care settings. Nearly all patients expressed at least one need for survivorship care, and in general, patients in primary care expressed more needs than patients in hospital. Furthermore, the study suggests that the association between gender and stated needs depends on the healthcare setting, that married and cohabiting patients state fewer needs, and that higher socioeconomic status in relation to educational level, income, and labor market status is associated with fewer stated needs. This emphasizes the need to systematically undertake needs assessments in hospitals and primary care settings in relation to survivorship care. A systematic assessment of needs will ensure that all patients receive an assessment of survivorship care needs and a referral if necessary. Future research should focus on exploring the patients' experiences with the process and involvement in the assessment of needs for survivorship care, which could expand the contextual understanding of the formal needs assessment.

\section{Acknowledgments}

We would like to thank the patients who participated in this study and made it possible. We would also like to thank the management and the staff for their generous contributions and kind assistance.

\section{Financial Disclosure}

This study was supported by the foundation "Folkesundhed $\mathrm{i}$ Midten", Denmark.

\section{Conflict of Interest}

The authors all declare that they have no competing interest and no potential conflict of interest with respect to the research, authorship, and/or publication of this article.

\section{Informed Consent}

Both oral and written consents from the patients were obtained.

\section{Author Contributions}

$\mathrm{CH}$ and TM contributed substantially to the conception and design of the study. CH, MLS, and TM analyzed and interpreted the data. $\mathrm{CH}$ drafted the manuscript, and $\mathrm{CH}, \mathrm{MLS}$, and TM all revised the manuscript critically for important intellectual content and gave final approval of the version to be published.

\section{References}

1. Dalton SO, Johansen C. New paradigms in planning cancer rehabilitation and survivorship. Acta Oncol. 2013;52(2):191-194.

2. Sandager M, Sperling C, Vinter MM, Lehmann Knudsen J, Jensen H. Cancer survivors' needs and experiences through treatment and subsequent. [Kraeftpatienters oplevelser med sundhedsvaesenet gennem udredning og behandling]. 2011.

3. Kjaer TK, Johansen C, Ibfelt E, Christensen J, Rottmann $\mathrm{N}$, Hoybye MT, Ross L, et al. Impact of symptom burden on health related quality of life of cancer survivors in a Danish cancer rehabilitation program: A longitudinal study. Acta Oncol. 2011;50(2):223-232.

4. Holm LV, Hansen DG, Johansen C, Vedsted P, Larsen PV, Kragstrup J, Sondergaard J. Participation in cancer rehabilitation and unmet needs: a population-based cohort study. Support Care Cancer. 2012;20(11):2913-2924.

5. Handberg C, Jensen CM, Maribo T. Lack of needs assessment in cancer survivorship care and rehabilitation in hospitals and primary care settings. J Clin Med Res. 2017;9(10):864-871.

6. World Health Organization (WHO). World report on Disability. 2011. http://whqlibdoc.who.int/publications/2011/9789240685215_eng.pdf?ua=1.

7. Johansen JS, Rahbek J, Moller K, Jensen L. The white paper - rehabilitation in Denmark [Rehabilitering i Danmark - Hvidbog om rehabiliteringsbegrebet]. MarselisborgCentret, Rehabiliteringsforum Danmark og MarselisborgCentret. 2004. http://www.rehabiliteringsforum.dk/ 
contentassets/519c219e42a3454da34db60a8454de58/ hvidbog.pdf.

8. Alfano CM, Ganz PA, Rowland JH, Hahn EE. Cancer survivorship and cancer rehabilitation: revitalizing the link. J Clin Oncol. 2012;30(9):904-906.

9. Budhwani S, Wodchis WP, Zimmermann C, Moineddin R, Howell D. Self-management, self-management support needs and interventions in advanced cancer: a scoping review. BMJ Support Palliat Care. 2019;9(1):12-25.

10. Friis K, Larsen FB, Nielsen CV, Momsen AH, Stapelfeldt CM. Social inequality in cancer survivors' health behaviours-A Danish population-based study. Eur J Cancer Care (Engl). 2018;27(3):e12840.

11. Holm LV, Hansen DG, Larsen PV, Johansen C, Vedsted P, Bergholdt SH, Kragstrup J, et al. Social inequality in cancer rehabilitation: a population-based cohort study. Acta Oncol. 2013;52(2):410-422.

12. Moustsen IR, Larsen SB, Vibe-Petersen J, et al. Social position and referral to rehabilitation among cancer patients. Acta Oncol. 2015;54(5):1-7.

13. Hansen DG, Larsen PV, Holm LV, Rottmann N, Bergholdt SH, Sondergaard J. Association between unmet needs and quality of life of cancer patients: a populationbased study. Acta Oncol. 2013;52(2):391-399.

14. Temel JS, Greer JA, El-Jawahri A, Pirl WF, Park ER, Jackson VA, Back AL, et al. Effects of early integrated palliative care in patients with lung and GI cancer: a randomized clinical trial. J Clin Oncol. 2017;35(8):834-841.

15. Salakari MR, Surakka T, Nurminen R, Pylkkanen L. Effects of rehabilitation among patients with advances cancer: a systematic review. Acta Oncol. 2015;54(5):618628.

16. Danish Health and Medicines Authority. Disease management program for rehabilitation and palliation in cancer: part of the overall disease management program for cancer [Forloebsprogram for rehabilitering og palliation i forbindelse med kraeft]. 2012.

17. Close Health Offers, Strategy and Planning, Central Denmark Region [Naere Sundhedstilbud, Strategi og Planlaegning, Region Midtjylland]. Health Agreement for rehabilitation of cancer, Health Agreement 2015-2018, Regional guideline [Sundhedsaftale om rehabilitering $\mathrm{i}$ forbindelse med kraeft, Sundhedsaftale 2015-2018, regional retningslinje]. 2015.

18. Handberg C, Thorne S, Maribo T. When a policy decision meets practice realities: The case of cancer survivorship care and rehabilitation needs assessment. Eur J Oncol Nurs. 2018;33:70-77.

19. Frank L. When an entire country is a cohort. Science. 2000;287(5462):2398-2399.

20. Baadsgaard M, Quitzau J. Danish registers on personal income and transfer payments. Scand J Public Health. 2011;39(7 Suppl):103-105.

21. Jensen VM, Rasmussen AW. Danish Education Registers. Scand J Public Health. 2011;39(7 Suppl):91-94.

22. Petersson F, Baadsgaard M, Thygesen LC. Danish registers on personal labour market affiliation. Scand J Public Health. 2011;39(7 Suppl):95-98.

23. Gjerstorff ML. The Danish Cancer Registry. Scand J Pub- lic Health. 2011;39(7 Suppl):42-45.

24. Klassen AC, Smith KC. The enduring and evolving relationship between social class and breast cancer burden: a review of the literature. Cancer Epidemiol. 2011;35(3):217-234.

25. Handberg C, Lomborg K, Nielsen CV, Oliffe JL, Midtgaard J. Understanding male cancer patients' barriers to participating in cancer rehabilitation. Eur J Cancer Care (Engl). 2015;24(6):801-811.

26. Peate I. Men and cancer: the gender dimension. Br J Nurs. 2011;20(6):340, 342-343.

27. Handberg C, Nielsen CV, Lomborg K. Men's reflections on participating in cancer rehabilitation: a systematic review of qualitative studies 2000-2013. Eur J Cancer Care (Engl). 2014;23(2):159-172.

28. Creighton G, Oliffe JL. Theorising masculinities and men's health: a brief history with a view to practice. Health Sociol Rev. 2010;19:409.

29. Courtenay WH. Constructions of masculinity and their influence on men's well-being: a theory of gender and health. Soc Sci Med. 2000;50(10):1385-1401.

30. White A, De Sousa B, De Visser R, et al. Men's health in Europe. J Women's Health. 2011;8(3):192.

31. Hunt K, Wyke S, Gray CM, Anderson AS, Brady A, Bunn $\mathrm{C}$, Donnan PT, et al. A gender-sensitised weight loss and healthy living programme for overweight and obese men delivered by Scottish Premier League football clubs (FFIT): a pragmatic randomised controlled trial. Lancet. 2014;383(9924):1211-1221.

32. Faller H, Koch U, Brahler E, Harter M, Keller M, Schulz $\mathrm{H}$, Wegscheider K, et al. Satisfaction with information and unmet information needs in men and women with cancer. J Cancer Surviv. 2016;10(1):62-70.

33. Street RL, Jr. Gender differences in health care providerpatient communication: are they due to style, stereotypes, or accommodation? Patient Educ Couns. 2002;48(3):201206.

34. Handberg C, Midtgaard J, Nielsen CV, Thorne S, Lomborg K. Healthcare Professionals' Attitudes to Rehabilitation Programming for Male Cancer Survivors. Rehabil Nurs. 2018;43(3):127-137.

35. Primeau SW, Freund KM, Ramachandran A, Bak SM, Heeren T, Chen CA, Morton S, et al. Social service barriers delay care among women with abnormal cancer screening. J Gen Intern Med. 2014;29(1):169-175.

36. Holm LV, Hansen DG, Kragstrup J, Johansen C, Christensen R, Vedsted P, Sondergaard J. Influence of comorbidity on cancer patients' rehabilitation needs, participation in rehabilitation activities and unmet needs: a population-based cohort study. Support Care Cancer. 2014;22(8):2095-2105.

37. Hoybye MT, Dalton SO, Christensen J, Ross L, Kuhn $\mathrm{KG}$, Johansen C. Social and psychological determinants of participation in internet-based cancer support groups. Support Care Cancer. 2010;18(5):553-560.

38. Johansen C, Dalton SO. Survivorship - searching for new directions. Acta Oncol. 2015;54(5):569-573.

39. The Danish Cancer Society [Kraeftens Bekaempelse]. From knowledge to action in cancer rehabilitation - Re- 
port from a National task group. [Fra viden til handling $i$ rehabiliteringsindsatsen i forbindelse med kraeft - Rapport fra en national arbejdsgruppe]. The Danish Cancer Society. 2015. Retrieved from http://www.cancer. $\mathrm{dk} / \mathrm{dyn} /$ resources/File/file/6/4676/1428488823/rapport-om-rehabilitering.-sammenfatning.-endelig-16.22015-national-arbejdsgruppe.pdf.

40. Temel JS, Greer JA, Muzikansky A, Gallagher ER, Admane S, Jackson VA, Dahlin CM, et al. Early palliative care for patients with metastatic non-small-cell lung cancer. N Engl J Med. 2010;363(8):733-742.

41. de Leeuw J, Larsson M. Nurse-led follow-up care for cancer patients: what is known and what is needed. Support Care Cancer. 2013;21(9):2643-2649.

42. Armes J, Crowe M, Colbourne L, Morgan H, Murrells T,
Oakley C, Palmer N, et al. Patients' supportive care needs beyond the end of cancer treatment: a prospective, longitudinal survey. J Clin Oncol. 2009;27(36):6172-6179.

43. Marengoni A, Angleman S, Melis R, Mangialasche F, Karp A, Garmen A, Meinow B, et al. Aging with multimorbidity: a systematic review of the literature. Ageing Res Rev. 2011;10(4):430-439.

44. Klein J, von dem Knesebeck O. Socioeconomic inequalities in prostate cancer survival: A review of the evidence and explanatory factors. Soc Sci Med. 2015;142:9-18.

45. Sleight AG, Lyons KD, Vigen C, Macdonald H, Clark F. The association of health-related quality of life with unmet supportive care needs and sociodemographic factors in low-income Latina breast cancer survivors: a singlecentre pilot study. Disabil Rehabil. 2018:1-6. 